\title{
Comparison of Uncertainty Quantification Approaches in a Supersonic Biplane Airfoil Problem*
}

\author{
Wataru YAMAZAKI $I^{\dagger}$ and Yuki SUGA \\ Department of Mechanical Engineering, Nagaoka University of Technology, Nagaoka, Niigata 940-2188, Japan
}

\begin{abstract}
In this paper, uncertainty quantification approaches are compared quantitatively in an aerodynamic uncertainty quantification problem for a 2D supersonic biplane airfoil. Three advanced uncertainty quantification approaches are compared: an inexpensive Monte-Carlo simulation approach using a Kriging response surface model, an intrusive polynomial chaos approach, and a point collocation non-intrusive polynomial chaos approach. Two-dimensional inviscid compressible flow around the supersonic biplane airfoil is considered with an uncertainty of the freestream Mach number as a normal distribution. A choking phenomenon occurs in this problem setting, which gives discontinuous changes in aerodynamic performance with fluctuation of the freestream Mach number. The accuracies and characteristics of the three uncertainty quantification approaches are investigated. The inexpensive Monte-Carlo simulation approach shows the best performance with larger numbers of sample points in this study. The results of the non-intrusive polynomial chaos approach are sensitive to sampling strategies. Although the intrusive polynomial chaos approach is applied only with lower orders of polynomial chaos, it shows comparable accuracy with the other two approaches from the viewpoint of model accuracy when weighted at the center region of the uncertain input space.
\end{abstract}

Key Words: Uncertainty Quantification, Polynomial Chaos, Monte-Carlo Simulation, Kriging Model, Supersonic Biplane Airfoil

\section{Introduction}

Uncertainty quantification (UQ) is being watched closely recently ${ }^{1-11)}$ since high-fidelity computational fluid dynamics (CFD) computations typically assume full knowledge of all parameters. In reality, there are many uncertainty sources, which are classified into "epistemic uncertainty" and "aleatory uncertainty" based on properties. Epistemic uncertainty is caused by a lack of knowledge or incomplete information on the behavior of a particular variable (e.g., empirically determined model coefficients). Aleatory uncertainty is, on the other hand, the inherent variation of a physical input parameter (e.g., uniform flow velocity magnitude). Such variation is due to the random nature of the input parameter and can be represented by a probability density function (PDF). For more advanced engineering design, it is therefore important to analyze the mean, standard deviation, PDF and cumulative distribution function (CDF) of performance functions with respect to uncertain input variables. One of the major applications of UQ in the aerodynamic design process is robust/reliability based design optimizations $^{12,13)}$ in which more robust/reliable optimal shapes can be designed. In these advanced design optimizations, variations in performance functions based on uncertain input parameters have to be evaluated accurately.

The most straightforward method for UQ is a full nonlinear Monte-Carlo simulation (FMCS). Although this method is easy to implement, it is still prohibitively expensive for

(C) 2017 The Japan Society for Aeronautical and Space Sciences

*Received 28 July 2015; final revision received 1 April 2016; accepted for publication 3 October 2016.

†Corresponding author, yamazaki@mech.nagaokaut.ac.jp high-fidelity CFD computations. To apply surrogate model approaches is another way for UQ, and this can reduce computational cost dramatically. This approach is often referred to as an inexpensive Monte-Carlo simulation (IMCS). ${ }^{7-9)}$ Polynomial chaos approaches $2,3,5,6,10,11,14)$ are other positive methods to model and propagate uncertainty in computational simulations. In the polynomial chaos approaches, uncertain variables are expanded to include random variables utilizing orthogonal polynomials. There are two variations of polynomial chaos approaches, often referred to as intrusive polynomial chaos (IPC) and non-intrusive polynomial chaos (NIPC). In the IPC approach, all dependent variables and uncertain input variables are replaced with their polynomial chaos expansions. Then, governing equations are expanded and UQ can be performed by solving the expanded governing equations directly. The NIPC approach is a kind of sampling based method and doesn't require any modifications in its original deterministic CFD code.

In this research, the three advanced UQ approaches (i.e., IMCS, IPC and NIPC) are compared in a UQ problem for a supersonic biplane airfoil. ${ }^{15)}$ We have recently developed an IPC approach for compressible inviscid flows and applied it to the UQ of a supersonic biplane airfoil. ${ }^{11)}$ It couldn't capture the effect of choking phenomena quantitatively, while the PDFs obtained showed qualitative agreements with those obtained using FMCS. To understand the details of the results obtained in the IPC analyses, and to compare with other UQ approaches, additional investigations are performed in this study. In the UQ problem, the input (aleatory) uncertainty is given for the freestream Mach number, and the propagation of uncertainty is discussed for the supersonic inviscid flow around the Busemann's biplane airfoil. This paper is 
organized as follows. A concise introduction of the UQ approaches is provided in Section 2. The UQ problem is outlined in Section 3. The detailed settings of the UQ approaches are explained in Section 4. The results of the UQ approaches are compared and discussed in Section 5, and concluding remarks are provided in Section 6.

\section{UQ Approaches}

In this section, uncertainty quantification approaches utilized in this research are concisely introduced. Readers can refer to Yamazaki ${ }^{10)}$ for the details of FMCS, IMCS and NIPC. The details of IPC are given in Suga and Yamazaki. ${ }^{11)}$

\subsection{FMCS}

In FMCS, a set of sample points for uncertain inputs is generated following the PDFs of the uncertain inputs. CFD evaluations are performed at the locations of sample points to calculate statistics directly. Although the FMCS approach is easy to implement, its computational cost is very large. The number of sample points for an accurate UQ is usually more than 1,000, which means 1,000 or more CFD functional evaluations are required to solve a UQ problem.

\subsection{IMCS}

The concept of IMCS is identical to that of FMCS. However, the huge number of exact functional evaluations is replaced by functional estimations on a surrogate model, which results in reducing the computational cost of UQ. In this research, an ordinary Kriging response surface model approach $^{9,16)}$ is utilized to construct a surrogate model in an uncertain input space. Then, Monte-Carlo simulation is performed on the surrogate model to calculate statistics. In this study, the number of sample points for IMCS is fixed at 10,000 .

\subsection{IPC}

Polynomial chaos is a stochastic method based on the spectral representation of uncertainty. ${ }^{2,14)}$ Uncertain variables are represented by a series of orthogonal polynomials as follows:

$$
w(\overrightarrow{\boldsymbol{x}}, \vec{\xi})=\sum_{i=0}^{Q} w_{i}(\overrightarrow{\boldsymbol{x}}) \Psi_{i}(\vec{\xi})
$$

where $w_{i}$ is the polynomial coefficient and $\Psi_{i}$ is the polynomial chaos basis of the $i$-th mode. $\overrightarrow{\boldsymbol{x}}$ and $\vec{\xi}$ respectively represent the vectors of deterministic variables and random variables. There is a one-to-one correspondence between a component of $\vec{\xi}$ and an uncertain input. $Q+1$ is the total number of terms which is defined as follows:

$$
Q+1=\frac{(n+p) !}{n ! p !}
$$

where $n$ and $p$ are respectively the number of uncertain inputs and the order of polynomial chaos expansion. $p$ determines the degree of freedom of uncertain variables so that complicated PDF can be expressed with a larger $p$. The choice of the polynomial chaos basis depends on the distribution of uncertain inputs. The optimal basis for a random distribution is found in the Askey scheme ${ }^{17)}$ (e.g., Hermite polynomial for Gaussian random distributions and Legendre polynomial for uniform random distributions). The detailed formulations are given in Yamazaki. ${ }^{10)}$ The ensemble average of the basis functions can be defined as:

$$
\left\langle\Psi_{i}(\vec{\xi}) \Psi_{j}(\vec{\xi})\right\rangle=\int \Psi_{i}(\vec{\xi}) \Psi_{j}(\vec{\xi}) W(\vec{\xi}) \mathrm{d} \vec{\xi}
$$

where $W(\vec{\xi})$ is the weight function corresponding to the type of the polynomial chaos basis. There is an orthogonal property of the polynomial chaos basis, which can be expressed as:

$$
\left\langle\Psi_{i}(\vec{\xi}) \Psi_{j}(\vec{\xi})\right\rangle=\left\langle\Psi_{i}^{2}\right\rangle \delta_{i j}
$$

where $\delta_{i j}$ is the Kronecker delta. In the IPC approach, all uncertain inputs and variables are expanded by Eq. (1), and then governing equations are expanded with a Galerkin projection using the relationships of Eqs. (3) and (4). Finally, the number of governing equations is increased from $n_{e q}$ (original number) to $n_{e q}(Q+1)$, and then all polynomial coefficients can be calculated by solving the expanded governing equations directly. $\left.{ }^{2}\right)$

\subsection{NIPC}

In the NIPC approaches, the polynomial coefficients $w_{i}$ are estimated without modifying the governing equations. In this research, a point-collocation NIPC approach ${ }^{5,6)}$ is utilized. In this approach, the polynomial coefficients are estimated by solving a system of equations constructed from a number of deterministic computational results $m . m$ vectors (samples) of $\vec{\xi}_{i}(i=1, \cdots, m)$ are chosen in the random space, and then $m$ deterministic computations are executed to obtain $w\left(\vec{\xi}_{i}\right)$. Finally a linear system of equations can be constructed from Eq. (1) as:

$$
\begin{gathered}
{\left[\begin{array}{cccc}
\Psi_{0}\left(\vec{\xi}_{1}\right) & \Psi_{1}\left(\vec{\xi}_{1}\right) & \ldots & \Psi_{Q}\left(\vec{\xi}_{1}\right) \\
\Psi_{0}\left(\vec{\xi}_{2}\right) & \Psi_{1}\left(\vec{\xi}_{2}\right) & \ldots & \Psi_{Q}\left(\vec{\xi}_{2}\right) \\
\vdots & \vdots & \ddots & \vdots \\
\Psi_{0}\left(\vec{\xi}_{m}\right) & \Psi_{1}\left(\vec{\xi}_{m}\right) & \ldots & \Psi_{Q}\left(\vec{\xi}_{m}\right)
\end{array}\right]\left[\begin{array}{c}
w_{0} \\
w_{1} \\
\vdots \\
w_{Q}
\end{array}\right]} \\
=\left[\begin{array}{c}
w\left(\vec{\xi}_{1}\right) \\
w\left(\vec{\xi}_{2}\right) \\
\vdots \\
w\left(\vec{\xi}_{m}\right)
\end{array}\right]
\end{gathered}
$$

The polynomial coefficients can be evaluated by solving the linear system of Eq. (5). Although $m$ can be defined arbitrarily, its recommended value is defined in Hosder et al. ${ }^{6}$ as:

$$
m=2(Q+1)
$$

In this case, an over-determined system of equations, Eq. (5), is solved using the Moore-Penrose pseudo-inverse matrix approach in the least-squares sense. Once all of the polynomial coefficients are determined using the IPC or NIPC methods, the mean and standard deviation of an uncertain variable can 
be calculated as:

$$
\begin{gathered}
\mu(w)=w_{0} \\
\sigma^{2}(w)=\sum_{i=1}^{Q} w_{i}^{2}\left\langle\Psi_{i}^{2}\right\rangle
\end{gathered}
$$

PDF as well as CDF of $w$ can also be evaluated inexpensively using the Monte-Carlo simulation for $\vec{\xi}$ in Eq. (1). In the case of $n=1$, the one-to-one correspondence between $\xi$ and an uncertain input $w_{i n}$, which has a normal distribution, is described as:

$$
w_{\text {in }}=\mu_{w_{\text {in }}}+\sigma_{w_{\text {in }}} \xi
$$

where $\mu_{w_{i n}}$ and $\sigma_{w_{i n}}$ are respectively the mean and standard deviation values of the uncertain input $w_{i n}$. From a different perspective, the polynomial chaos approach is therefore considered as a kind of surrogate model approach, since the functional variation with respect to $w_{\text {in }}$ can be estimated in Eq. (1) using the parameter $\xi$.

\section{UQ Problem of Supersonic Biplane Airfoil}

In this research, supersonic inviscid flows around the Busemann's biplane airfoil are analyzed. The thickness ratios of both airfoils are 0.05 . The distance ratio between the airfoils is set to 0.5 . The angle of attack is set to 2 degrees. The input (aleatory) uncertainty is given on the freestream Mach number as a normal distribution. Its mean and standard deviation values are respectively set to 1.7 and 0.0333 $\left(M_{\infty}=N\left(1.7,0.0333^{2}\right)\right)$.

Two-dimensional compressible Euler equations are solved using a gridless method ${ }^{18,19)}$ in this research. The flow flux at the intermediate position of two computational points is evaluated by the approximate Riemann solver of Roe with second-order spatial accuracy. The temporal discretization is treated using a four-stage Runge-Kutta scheme. Expanded governing equations obtained in the IPC approach are also solved using the gridless method.

Deterministic computational results in the range of $1.55<M_{\infty}<1.85$ are shown in Fig. 1 with the PDF of $M_{\infty}$. In Fig. 2, (deterministic) static pressure distributions at the $M_{\infty}$ of $1.6,1.7$ and 1.8 are visualized, clearly indicating choking phenomenon at a $M_{\infty}$ of 1.6. This phenomenon causes massive changes in the lift and drag coefficients as shown in Fig. 1. In detail, the choking phenomenon occurs at $M_{\infty}<1.615$, which means the probability of choking in the present UQ problem is about $0.5 \%$. Although the present UQ problem has only one uncertain input variable, this is a very practical and complicated problem due to the choking phenomenon.

\section{Implementation Details}

\subsection{FMCS}

A FMCS analysis is performed using 250 sample points for comparison. This means 250 deterministic CFD computations with different freestream Mach numbers are required for the FMCS analysis. The distribution of the 250 points is

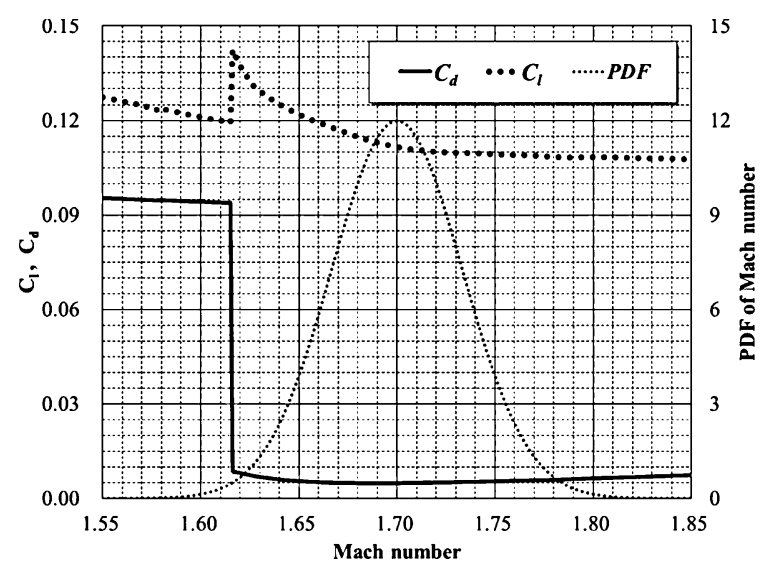

Fig. 1. Aerodynamic performance of Busemann's biplane airfoil with respect to the freestream Mach number.
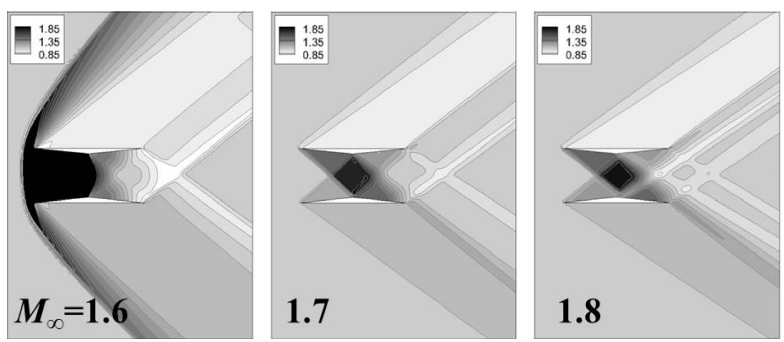

Fig. 2. Pressure variation around Busemann's biplane airfoil.

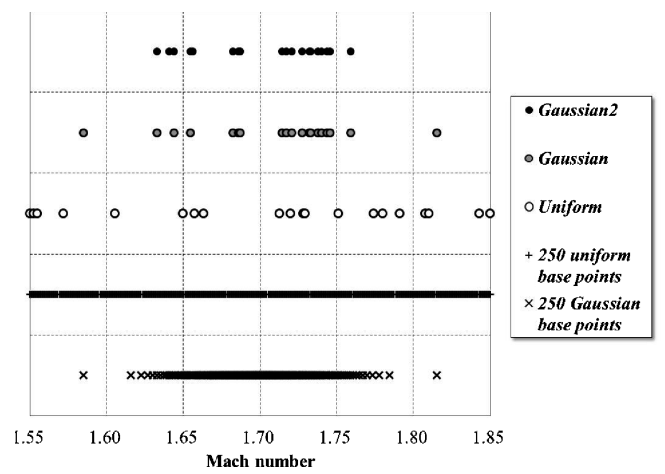

Fig. 3. Comparison of three sampling strategies selecting 20 sample points.

shown in the lowest part of Fig. 3 (i.e., also used as the base points for IMCS and NIPC). In the 250 samples, the choking phenomenon occurs only on a sample point, at a $M_{\infty}$ of 1.585. Please note that this FMCS result shouldn't be considered the "correct" solution of this UQ problem (i.e., 250 sample points are not enough for accurate FMCS).

\subsection{IMCS}

In the IMCS analysis, the number of sample points to construct a Kriging response surface model can be changed arbitrarily. Three sampling strategies are examined in this study: Uniform, Gaussian and Gaussian2. First, 250 uniform and Gaussian base sample points are generated uniformly in the range of $1.55<M_{\infty}<1.85$ or generated following the PDF of $M_{\infty}$. In the Uniform strategy, sample points are selected uniformly from the 250 uniform base sample points. In the Gaussian strategy, both end points (at locations ap- 
proximately $\pm 3.5 \sigma$ ) are first picked up, and then the remaining points are selected randomly from the remaining 248 Gaussian base sample points. In the Gaussian 2 strategy, both end points are never picked up, and all points are selected randomly from the remaining 248 Gaussian base sample points. In Fig. 3, the distributions of the 250 base points are indicated. In addition, sample points obtained by the three strategies are compared in Fig. 3, in which 20 sample points are selected following the above principles.

\subsection{IPC}

In the IPC analysis, the order of polynomial chaos $p$ can be changed arbitrarily. In this study, the conditions of $p=1,2$ are considered. ${ }^{11)}$ As reference information, deterministic CFD computations required in other UQ approaches were performed with this IPC program code by setting $p=0$, so there is no variance between the IPC approach and other UQ approaches. The computational costs of the IPC approaches were approximately $2(p=1)$ and $4(p=2)$ taking the computational cost of a deterministic CFD computation as one unit.

\subsection{NIPC}

In the NIPC analysis, the order of polynomial chaos $p$ as well as the number of sample points $m$ can be changed arbitrarily. The sample points are selected in the same manner as described in Sub-section 4.2. The order of polynomial chaos is changed from 1 to 20 in this study.

\section{Results and Discussion}

We have compared the performance of UQ approaches in lift coefficient, drag coefficient and $L / D$. Since the tendencies were almost comparable in all of the performance functions, the results of drag coefficient are mainly shown in this paper.

\subsection{Results of IMCS}

Surrogate models obtained using the three sampling strategies are compared in Fig. 4 with the number of sample points being 200. In the Kriging response surface model approach, estimated functional values perfectly agree with the exact functional values at the sample points locations, so uniform sampling provided the most accurate surrogate model over the entire uncertain input space. The other two strategies showed less accuracy around both ends of the uncertain input space due to the smaller number of sample points. Since this tendency was the same for the cases with other numbers of sample points, only the IMCS results with uniform sampling are shown hereafter. The IMCS results with larger numbers of sample points using uniform sampling can be considered the most exact estimates in this research.

\subsection{Comparison of statistics}

The mean and standard deviation values of $C_{d}$ obtained from the FMCS, IMCS, IPC and NIPC approaches are compared in Figs. 5-7. In these figures, FMCS results should not be considered as correct solutions. In our opinion, IMCS results with larger numbers of sample points should be considered to be the most accurate results in these figures in view of the accurate surrogate models, as indicated in Fig. 4. The

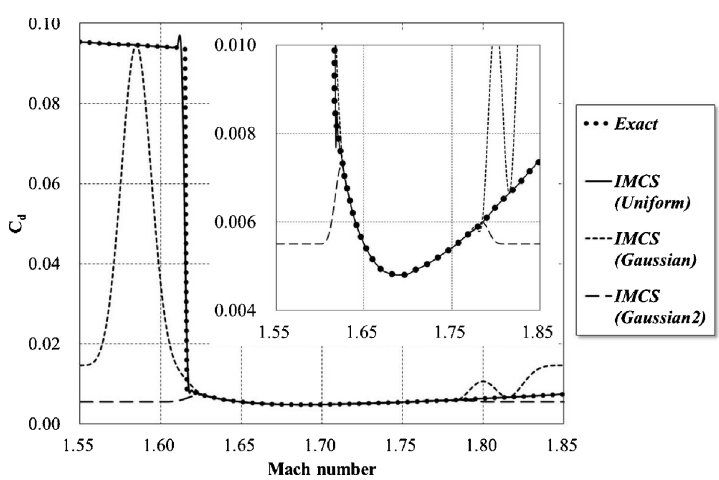

Fig. 4. Kriging models of $C_{d}$ constructed with three different sampling strategies.
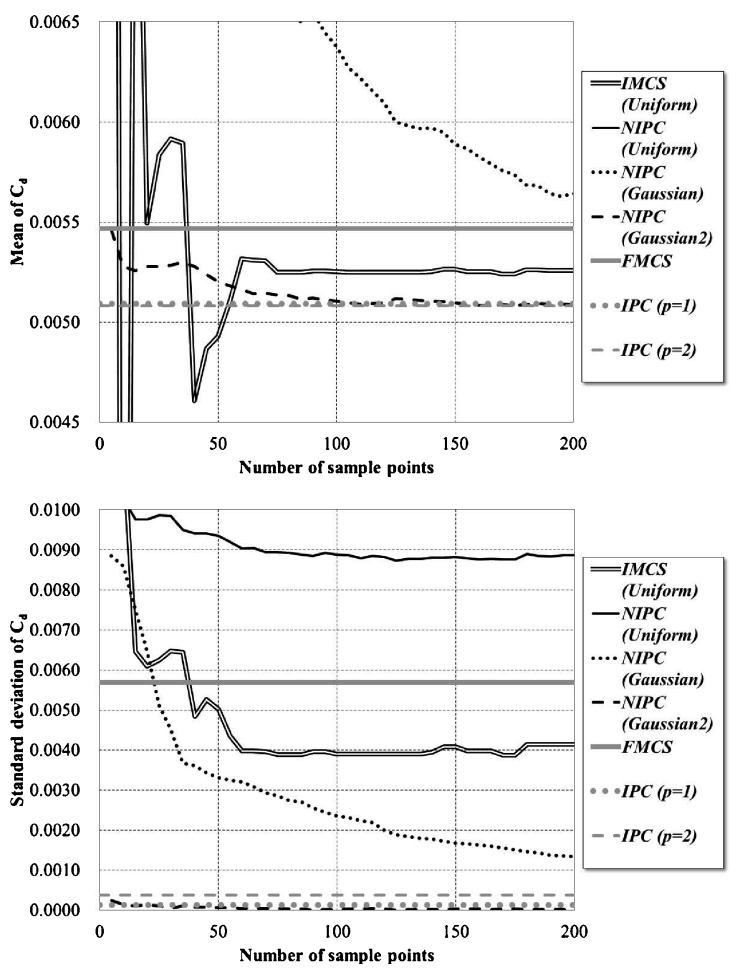

Fig. 5. Variation of statistics at $p=1$ in NIPC, Upper: mean, Lower: standard deviation of $C_{d}$.

standard deviation values obtained using IPC are very different from those obtained using FMCS and IMCS. This is due to the influence of not capturing the choking phenomena in the IPC approaches, which was discussed in Suga and Yamazaki. ${ }^{11)}$ It can be seen that the NIPC results are very different depending on the sampling strategies, even at a same set of $p$ and $m$. On the other hand, the IPC and NIPC (Gaussian2) results show qualitative agreements at larger $m$ as understood from the data of Figs. 5 and 6. With larger $p$, NIPC (Uniform) is considered to be the most accurate in the NIPC results since it qualitatively agrees with the IMCS results at larger $m$ (lines of the other two NIPC approaches are out of range in the lower graph of Fig. 7). With smaller $p$, on the other hand, the other two NIPC approaches show relatively better performance, especially in the graphs of the mean values (The line of NIPC (Uniform) is out of range in the upper graph of Fig. 5). 

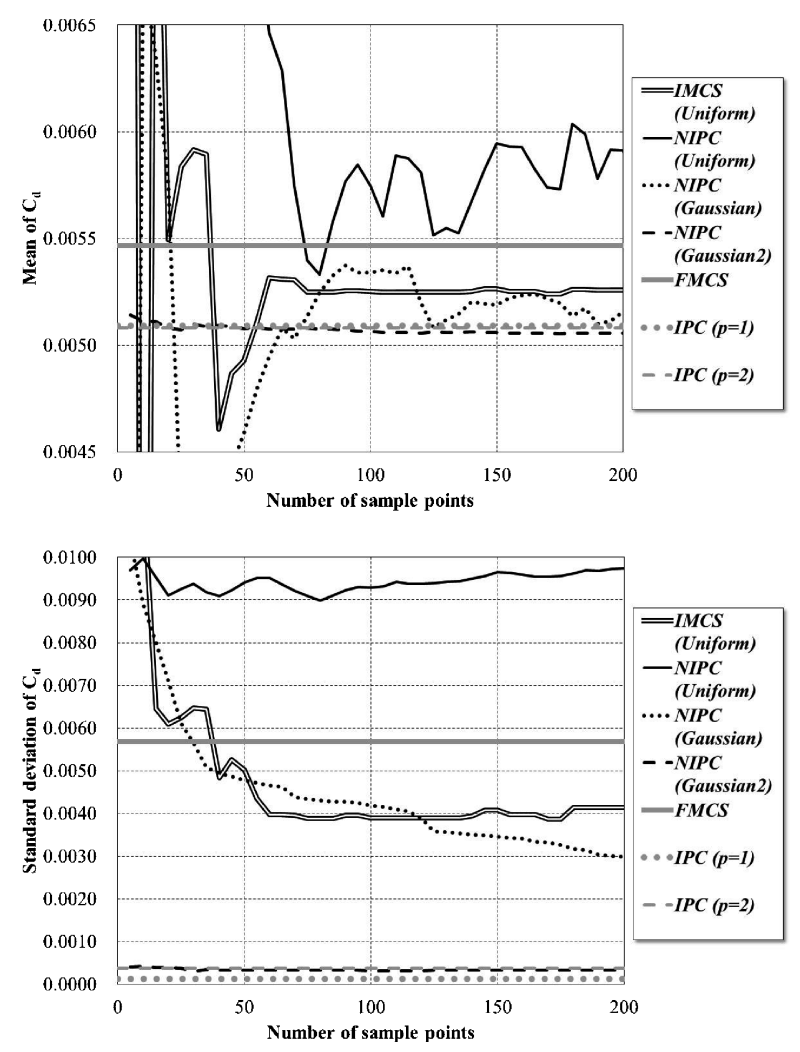

Fig. 6. Variation of statistics at $p=2$ in NIPC, Upper: mean, Lower: standard deviation of $C_{d}$.
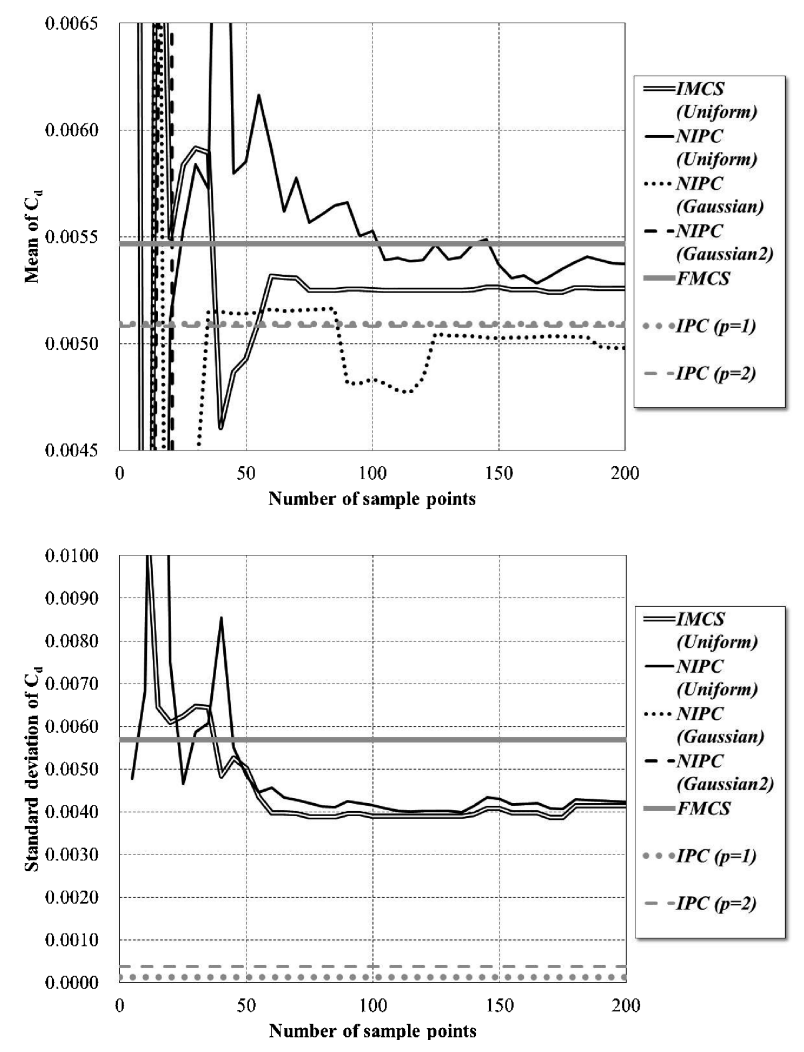

Fig. 7. Variation of statistics at $p=20$ in NIPC, Upper: mean, Lower: standard deviation of $C_{d}$.

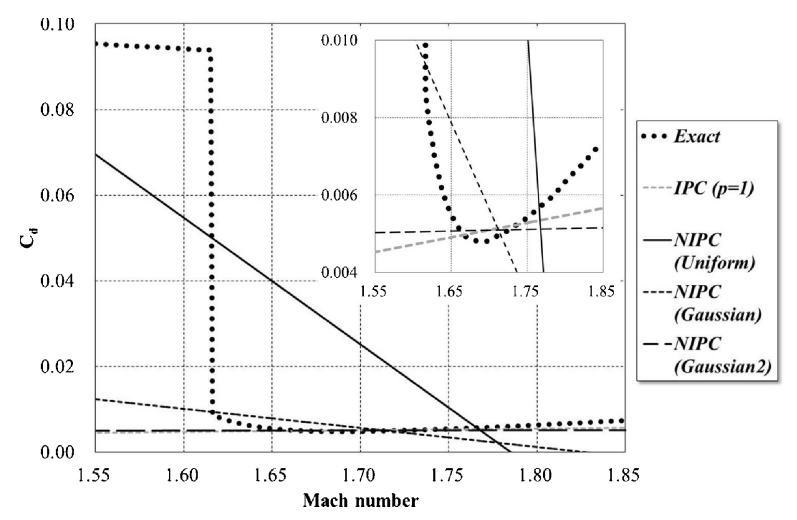

Fig. 8. Surrogate models of $C_{d}$ at $p=1$.

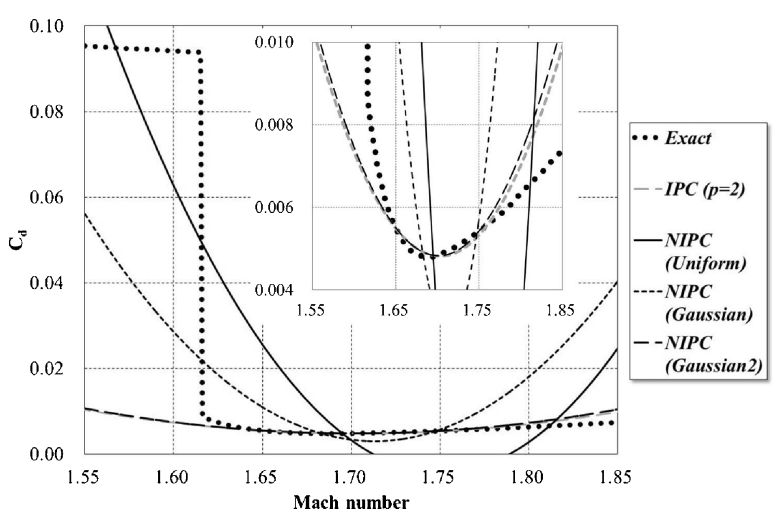

Fig. 9. Surrogate models of $C_{d}$ at $p=2$.

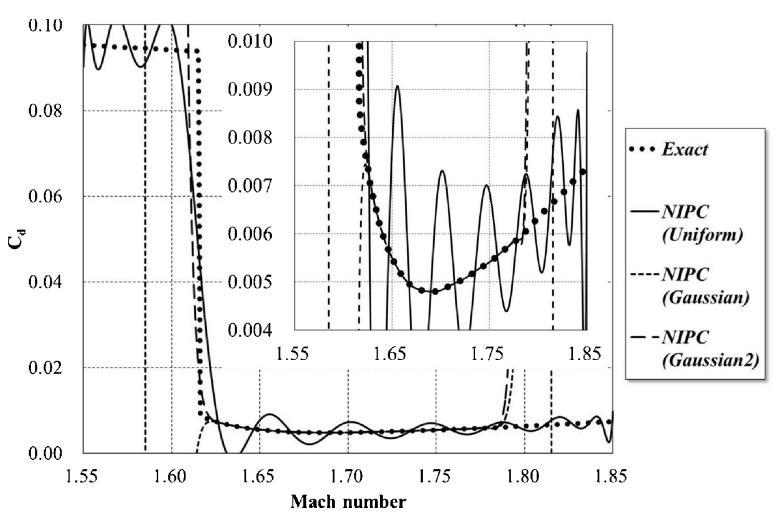

Fig. 10. Surrogate models of $C_{d}$ at $p=20$ in NIPC.

\subsection{Visualization of surrogate models}

As explained in Sub-section 2.4, the polynomial chaos can be considered a kind of surrogate model approach. Visualization of the surrogate models in the polynomial chaos approaches is performed in this sub-section to provide a detailed discussion about the results of the previous subsection.

In Figs. 8-10, the NIPC surrogate models obtained with an $m$ of 200 are compared with those of the IPC approaches. Since the polynomial coefficients are determined in the leastsquares sense in the NIPC approaches, the estimated functional values don't agree with the exact functional values at the sample point locations. At a $p$ of 1 , the surrogate models are linear functions since it has the lowest degree 
of freedom to express uncertainty, while surrogate models are parabolic functions at a $p$ of 2. The NIPC (Uniform) model is very different from the others because it takes into account a larger number of sample points located in the choking region $\left(M_{\infty}<1.615\right)$; especially at a $p$ of 1 . This is considered the reason why the statistics in NIPC (Uniform) are relatively inaccurate at a lower $p$. By considering the choking effect within the lower degree of freedom to express uncertainty, surrogate models degenerate, which results in the inaccuracy of estimated statistics. IPC models are comparable to the NIPC (Gaussian2) models at both $p$ of 1 and 2. The complicated behaviors of $C_{d}$ are better expressed with larger $p$ as shown in Fig. 10. Although the NIPC models (Gaussian and Gaussian2) agree well with the exact data in the region of $1.625<M_{\infty}<1.775$, the estimations of these models in the outer regions are out of range. The NIPC (Uniform) models showed reasonable estimations over the entire region at a $p$ of 20 while oscillations of the model are also observed. This is considered to be the reason why the NIPC (Uniform) statistics are relatively accurate with a larger $p$. No or little consideration about the choking phenomenon in NIPC formulations with a larger $p$ results in ridiculous estimations in the regions.

\subsection{Model accuracy}

From the discussion in the previous sub-section, two measures to evaluate the accuracy of surrogate models quantitatively in this UQ problem can be defined. The first measure is the overall model accuracy. The other measure is the weighted model accuracy in which the model accuracy for the center region (mean location) is emphasized. In this research, the following two measures are defined:

$$
\begin{aligned}
E & =\frac{1}{0.3} \int_{1.55}^{1.85}\left|C_{d}^{\text {exact }}-C_{d}^{\text {estimated }}\right| \mathrm{d} M \\
& \cong \frac{1}{250} \sum_{i=1}^{250}\left|C_{d i}^{\text {exact }}-C_{d i}^{\text {estimated }}\right| \\
E_{w} & =\int_{1.55}^{1.85}\left|C_{d}^{\text {exact }}-C_{d}^{\text {estimated }}\right| \phi \mathrm{d} M \\
& \cong \frac{0.3}{250} \sum_{i=1}^{250}\left|C_{d i}^{\text {exact }}-C_{d i}^{\text {estimated }}\right| \phi_{\left(M_{i}\right)}
\end{aligned}
$$

where $\phi$ is the PDF of $M_{\infty}$. Here, the exact CFD data of the 250 uniform base sample points described in Sub-section 4.2 are utilized to evaluate the measures. $E$ corresponds to the overall model accuracy while $E_{w}$ corresponds to the weighted model accuracy.

The two measures are evaluated in the surrogate models of the NIPC approaches with variations in $m$ and $p$. The results are summarized in the color maps of Fig. 11. The color maps show clear differences in the sampling strategies. With respect to $E$, more accurate models are obtained for the uniform sampling with a larger $p$, while worse models are obtained for the Gaussian/Gaussian2 samplings with a larger $p$. With respect to $E_{w}$, more accurate models are obtained for the uniform sampling with a larger $p$, while much more accurate models are obtained for the Gaussian/Gaussian2 samplings with a relatively small $p$. For a detailed discussion about
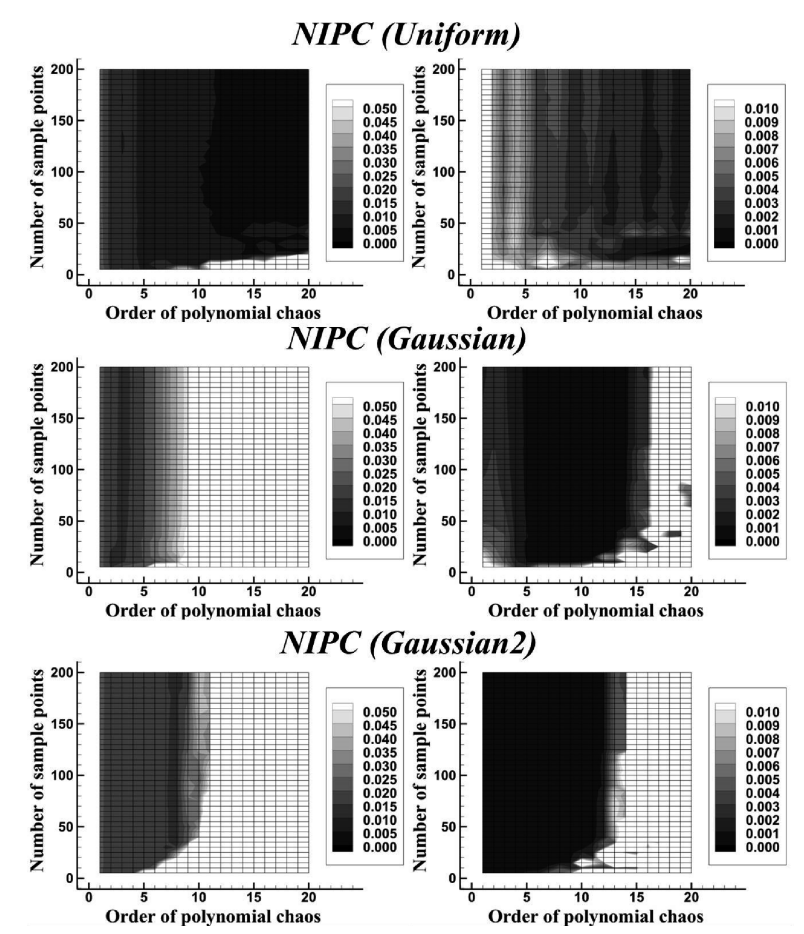

Fig. 11. Maps of two measures of model accuracy, Left: $E$, Right: $E_{w}$.
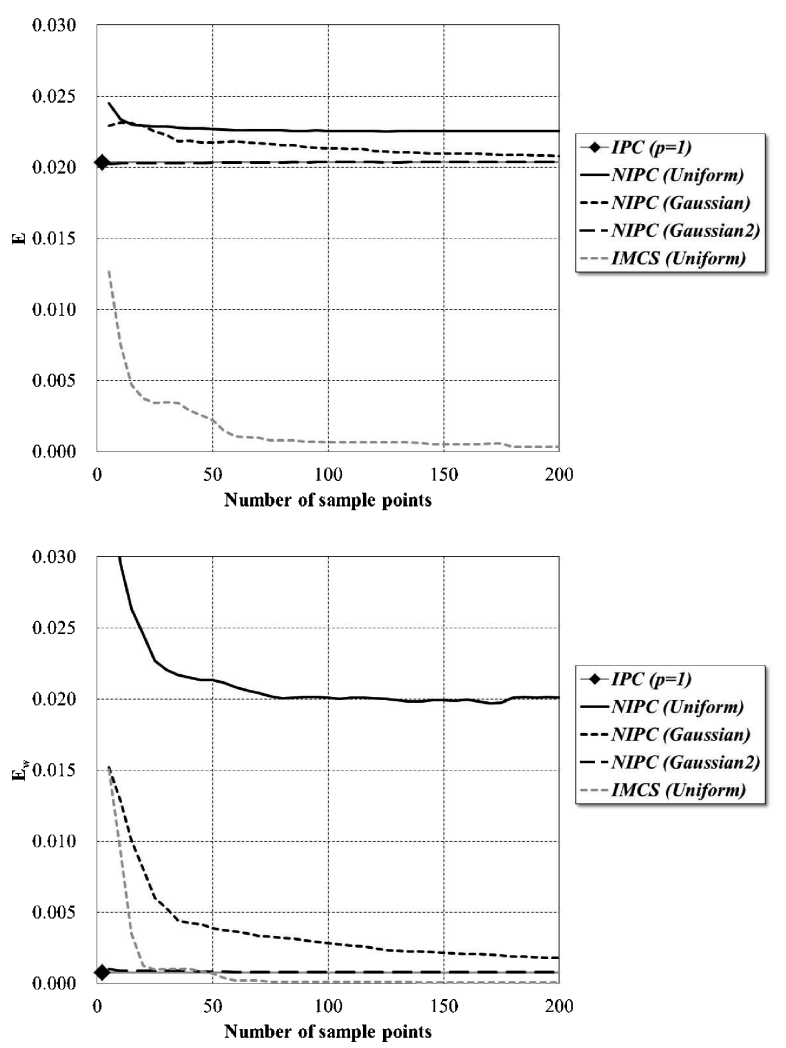

Fig. 12. Variation in model accuracy measures at $p$ of 1, Upper: $E$, Lower: $E_{w}$.

the IPC results, the variations of $E$ and $E_{w}$ at a $p$ of 1 and 2 are compared in Figs. 12 and 13. The performance of IMCS is also included in Figs. 12 and 13. The IMCS shows the best performance with larger numbers of sample points, from both viewpoints of $E$ and $E_{w}$. The performance of the IPC approaches is almost comparable to that of NIPC 

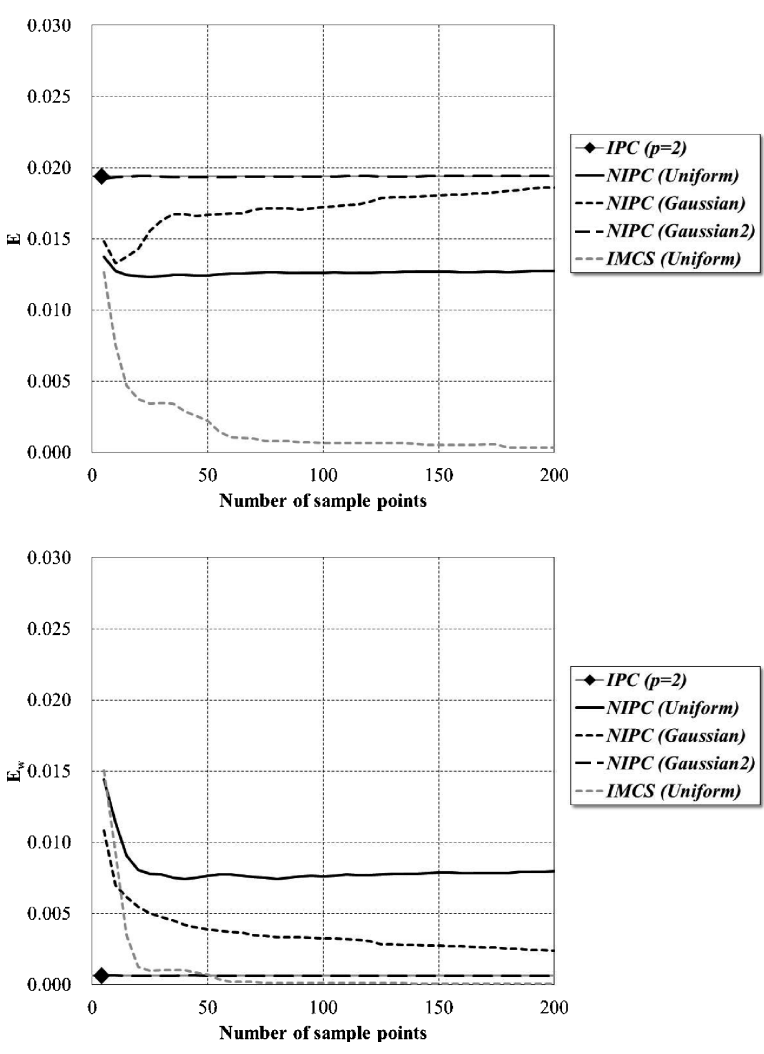

Fig. 13. Variation in model accuracy measures at $p$ of 2, Upper: $E$, Lower: $E_{w}$.

(Gaussian2), showing good accuracy from the viewpoint of $E_{w}$. Since the $x$-axis of Figs. 12 and 13 is equivalent to the computational cost for UQ analysis (i.e., computational cost of IMCS/NIPC itself is much smaller than that for the evaluations of sample points), diamond shapes are plotted in the figures at the corresponding computational costs of the IPC approaches. It can be seen that the IPC approaches provide very accurate results from the viewpoint of $E_{w}$, having much smaller computational cost than the other approaches. Although the performance of the NIPC approaches is sensitive to the sampling strategies, the IPC approach can provide consistently accurate results from the viewpoint of $E_{w}$, which is considered a benefit of the IPC approach.

In practical engineering problems, quantitative evaluations of model accuracy are impractical, which means the number of sample points (and those locations) required for an accurate UQ cannot be determined beforehand in IMCS/NIPC approaches. In the IPC approach, on the other hand, reasonable predictions in terms of weighted model accuracy can be expected without any consideration of sample points.

\subsection{Comparison of CDF}

Finally, the $\mathrm{CDF}$ distributions of $C_{d}$ and $C_{l}$ obtained are compared in Figs. 14 and 15. In these figures, IMCS/NIPC results using 200 sample points were indicated. The CDFs obtained by the IPC approaches are much closer to that of FMCS/IMCS among the polynomial chaos approaches using the same set of $p$, which is clearly observable in $C_{d}$. The CDFs obtained using the IPC approach with a $p$ of 2 are very comparable to those of FMCS and IMCS, which in-
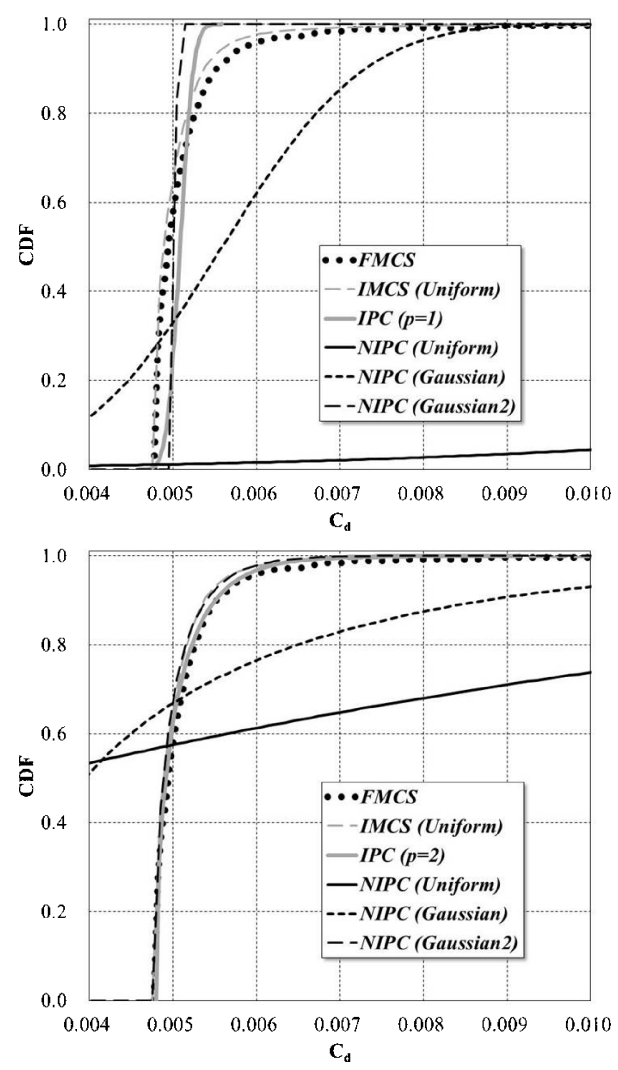

Fig. 14. Comparison of $\mathrm{CDF}$ of $C_{d}$ at $p=1$ (upper) and $p=2$ (lower).
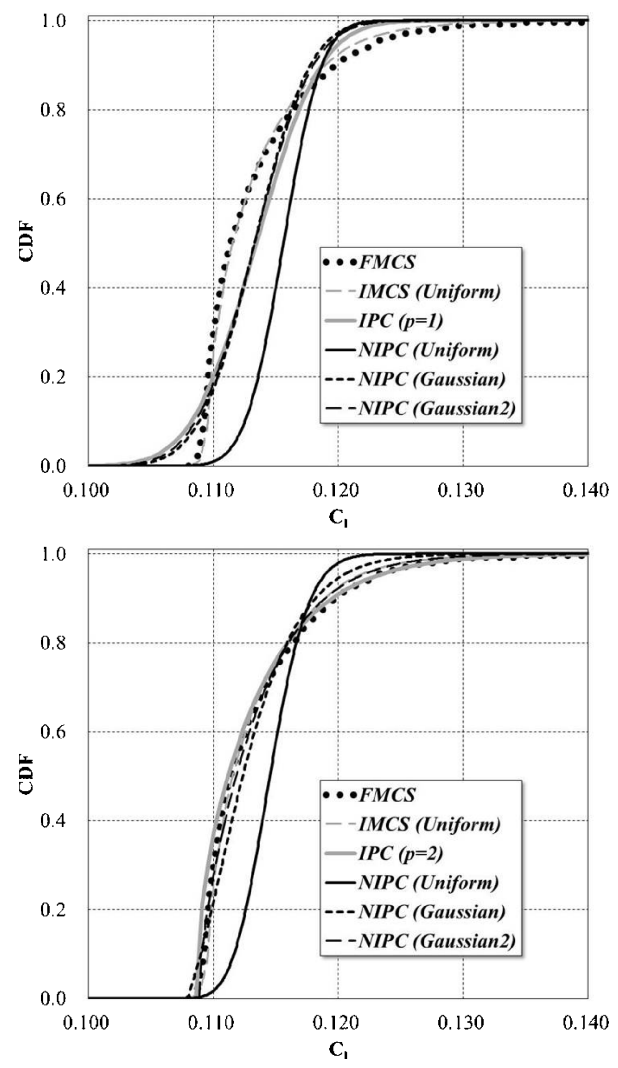

Fig. 15. Comparison of CDF of $C_{l}$ at $p=1$ (upper) and $p=2$ (lower). 
dicates the engineering availability of the UQ based on the IPC approach.

\section{Conclusions}

In this paper, several UQ approaches were compared applying an aerodynamic UQ problem of Busemann's biplane airfoil. The input uncertainty on the freestream Mach number is given as a normal distribution, and then the uncertainty propagation in the supersonic inviscid flow around the Busemann's biplane airfoil is discussed. Although there was only one uncertain input, the UQ problem was practical and complicated due to the effect of the choking phenomenon.

The most accurate UQ results were obtained using IMCS with a larger number of sample points. This is due to the flexibility of the Kriging response surface model in representing nonlinear aerodynamic functions. The NIPC results were sensitive to the strategy of sample point selection. In this study, the Gaussian samplings showed better accuracy at a lower $p$. At a larger $p$, on the other hand, uniform sampling showed better accuracy, but oscillations were observed in the surrogate models obtained. To evaluate the model accuracy quantitatively, overall and weighted model accuracy measures were defined. Those analyses clearly revealed the influence of the sampling strategies in the NIPC approaches. As a result, it was confirmed that the NIPC approach is very sensitive to the number of sample points and their locations. On the other hand, the IPC approach can be executed without any consideration of sample points. The IPC results were comparable to those of NIPC with the Gaussian2 sampling strategy in this study, which provided good accuracy from the viewpoint of the weighted model accuracy. Since the computational cost of the IPC approach is much smaller than the other UQ approaches, it can be concluded that the IPC approach is very useful, even with a lower $p$, from the viewpoints of computational cost and weighted model accuracy.

As future work, the authors will investigate in other UQ problems to obtain more universal knowledge of UQ approaches and to enhance the validity and generality of the present results and discussion. The proposal of better sampling strategies for NIPC is another future research topic.

\section{References}

1) Luckring, J. M., Hemsch, M. J., and Morrison, J. H.: Uncertainty in Computational Aerodynamics, AIAA Paper 2003-0409, 2003.
2) Xiu, D. and Karniadakis, G. E.: Modeling Uncertainty in Flow Simulations via Generalized Polynomial Chaos, J. Comput. Phys., 187 (2003), pp. 137-167.

3) Debusschere, B. J., Najm, H. N., Pebay, P. P., Knio, O. M., Ghanem, R. G., and Maitre, O. P. L.: Numerical Challenges in the Use of Polynomial Chaos Representations for Stochastic Processes, SIAM J. Sci. Comput., 26, 2 (2004), pp. 698-719.

4) Ghate, D. and Giles, M. B.: Inexpensive Monte Carlo Uncertainty Analysis, Recent Trends in Aerospace Design and Optimization, Tata McGraw-Hill, New Delhi, 2006, pp. 203-210.

5) Hosder, S., Walters, R. W., and Perez, R.: A Non-intrusive Polynomial Chaos Method for Uncertainty Propagation in CFD Simulations, AIAA Paper 2006-891, 2006.

6) Hosder, S., Walters, R. W., and Balch, M.: Efficient Sampling for NonIntrusive Polynomial Chaos Applications with Multiple Uncertain Input Variables, AIAA Paper 2007-1939, 2007.

7) Rumpfkeil, M. P., Yamazaki, W., and Mavriplis, D. J.: Uncertainty Analysis Utilizing Gradient and Hessian Information, Proceedings of 6th International Conference on Computational Fluid Dynamics, 2010.

8) Lockwood, B. A., Rumpfkeil, M. P., Yamazaki, W., and Mavriplis, D. J.: Uncertainty Quantification in Viscous Hypersonic Flows using Gradient Information and Surrogate Modeling, AIAA Paper 2011885, 2011.

9) Yamazaki, W. and Mavriplis, D. J.: Derivative-enhanced Variable Fidelity Surrogate Modeling for Aerodynamic Functions, AIAA J., 51, 1 (2013), pp. 126-137.

10) Yamazaki, W.: Stochastic Drag Analysis via Polynomial Chaos Uncertainty Quantification, Trans. Jpn. Soc. Aeronaut. Space Sci., 58, 2 (2015), pp. 89-99.

11) Suga, Y. and Yamazaki, W.: Aerodynamic Uncertainty Quantification of Supersonic Biplane Airfoil via Polynomial Chaos Approach, AIAA Paper 2015-1815, 2015.

12) Lee, K. H. and Park, G. J.: A Global Robust Optimization Using Kriging Based Approximation Model, JSME Int. J. Ser. C, 49, 3 (2006), pp. 779-788.

13) Lian, Y. and Kim, N. H.: Reliability-Based Design Optimization of a Transonic Compressor, AIAA J., 44, 2 (2006), pp. 368-375.

14) Wiener, N.: The Homogeneous Chaos, Am. J. Math., 60, 4 (1938), pp. 897-936.

15) Kusunose, K., Matsushima, K., and Maruyama, D.: Supersonic Biplane-A Review, Prog. Aerospace Sci., 47 (2011), pp. 53-87.

16) Jones, D. R., Schonlau, M., and Welch, W. J.: Efficient Global Optimization of Expensive Black-Box Functions, J. Global Optim., 13 (1998), pp. 455-492.

17) Askey, R. and Wilson, J.: Some Basic Hypergeometric Orthogonal Polynomials that Generalize Jacobi Polynomials, Memoirs of the American Mathematical Society, Vol. 54, No. 319, 1985.

18) Ma, Z., Chen, H., and Zhou, C.: A Study of Point Moving Adaptivity in Gridless Method, Comput. Methods Appl. Mech. Eng., 197, 21-24 (2008), pp. 1926-1937.

19) Safian, K. and Yamazaki, W.: Effect of Point Adaptation in Gridless CFD Method, Proceedings of 50th Aircraft Symposium, 2012 (in Japanese). 